\title{
Spin Mixing in Ferromagnets Revealed
}

\section{Experiments with thin magnetic films show that mixing between spin states has a larger than expected effect on spectroscopic measurements used to probe magnetic interactions in materials.}

\author{
By Andrew D. Kent
}

A common way to study magnetic systems is ferromagnetic resonance (FMR) spectroscopy, in which absorption features are studied under the presence of a magnetic field. Over 70 years ago, Charles Kittel worked out how to interpret FMR measurements [1]. Kittel's theory is part of the bedrock of today's magnetism research; it is used on a daily basis worldwide to extract information about atomic-scale magnetic interactions within a material. An important observational parameter from FMR experiments is the $g$ factor, which characterizes the spin and orbital contributions to a

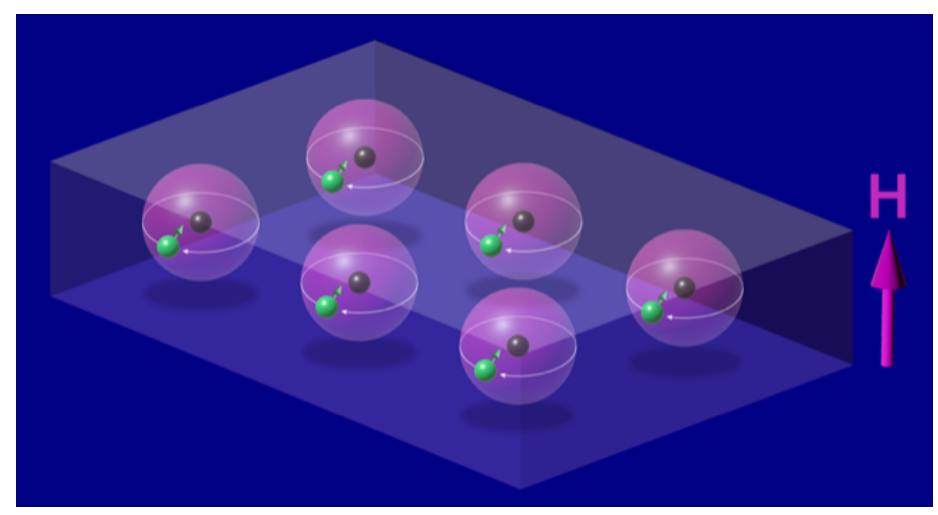

Figure 1: In a thin ferromagnetic film, the electrons' magnetic moment depends on both their spin and orbital motion. The ratio of orbital-to-spin magnetic moments has traditionally been measured with ferromagnetic resonance (FMR) spectroscopy. However, new experiments show that the basic theoretical model behind FMR needs to be updated to account for effects from spin mixing.

Credit: APS/Alan Stonebraker material's magnetic moment. Now, Justin Shaw from the National Institute of Standards and Technology (NIST), Colorado, and colleagues shows that the $g$ factor contains more information than Kittel originally accounted for [2]. Specifically, the $g$ factor can reveal the degree of spin mixing, which means the spin states of the electrons are mixtures of pure "up" and "down" (where up and down are relative to the magnetic field). Models of spintronic devices often ignore spin mixing by assuming that an electron is in a pure spin state. However, a better accounting of spin mixing-as provided by Shaw and colleagues-will help in describing spintronic phenomena, such as magnetic tunnel junctions [3], spin-transfer torques [4], and spin dynamics [5].

The dimensionless $g$ factor relates magnetic moment to angular momentum. For example, an electron's spin magnetic moment $\mu_{s}$ is related to its spin angular momentum by a $g$ factor that is approximately 2 (i.e., $g \cong 2$ ). In a condensed-matter system, electrons also have orbital angular momentum and a corresponding orbital magnetic moment $\mu_{L}$, which depends on the material's electronic structure. As Kittel showed, one can probe the orbital contribution to the magnetic moment of the electrons by measuring the $g$ factor with FMR spectroscopy. In this method, one places a sample in a magnetic field and measures the absorption of microwave frequency photons. The resonant absorption occurs when the photon energy is equal to the Zeeman splitting between adjacent electron eigenstates. When the magnetization is saturated and perpendicular to the sample plane, the slope of the resonance-frequency-applied field dispersion is proportional to the $g$ factor. According to Kittel's calculations, the $g$ factor is related to the ratio of 
orbital-to-spin magnetic moments through the expression $g=2+2 \mu_{L} / \mu_{S}$

Shaw and colleagues have now performed a critical experimental test of Kittel's $g$-factor expression using a combination of FMR and $x$-ray magnetic circular dichroism (XMCD) [2]. XMCD employs circularly polarized $x$ rays that are resonant with transitions in a material's atoms between inner (core) electron levels and outer (conduction) levels. Depending on their polarization, the $x$ rays excite electrons with spins oriented either nearly parallel or nearly antiparallel to the applied magnetic field. The difference in absorption of left-handed and right-handed circularly polarized $\mathrm{x}$ rays can be related to the ratio of orbital-to-spin magnetic moments in the material's conduction electrons $[6,7]$.

Shaw and colleagues performed XMCD on several ferromagnetic thin films, measuring their ratio of orbital-to-spin magnetic moments. They then conducted FMR spectroscopy on the same samples over a broad frequency range to determine the $g$ factor. Finally, the researchers compared the XMCD orbital-to-spin ratio to the ratio derived from FMR using Kittel's expression. The differences were both systematic and large, with the XMCD orbital-to-spin ratio being around 30\% larger than that found by FMR. These surprising results were consistent for all the materials tested, which included a series of magnetic multilayers (CoFe/Ni) and a film of permalloy $\left(\mathrm{Ni}_{80} \mathrm{Fe}_{20}\right)$, one of the most commonly studied transition-metal ferromagnetic alloys.

The fundamental question, of course, is why is there such a large discrepancy? The reason turns out to be an approximation used in Kittel's model. His theory only considered the lowest order effects of spin mixing by assuming that spin-mixed states are separated by the same amount of energy as pure spin states. This approximation was completely reasonable given the lack of precision of FMR spectroscopy when Kittel introduced his model. However, times have changed, and now the $g$ factor can be measured with a precision of less than $0.1 \%$ [8]. That higher precision means that one can start to probe higher-order effects of spin mixing. As Shaw and colleagues show, combining FMR with XMCD enables determination of the spin-mixing parameter, which characterizes how much of the down state, for example, is mixed with a nearly pure up state. This experimentally determined spin-mixing parameter is consistent with ab initio relativistic electronic structure calculations. The results show that even a small spin-mixing parameter $(\sim 2 \%)$ has a significant effect on FMR spectra.

These findings are important for several reasons. First, they demonstrate an experimental means for quantifying spin mixing in magnetic materials-something that wasn't available before. A second consequence is that spin mixing may be important more broadly to our understanding of spintronic and spin-transport phenomena. For example, a spin-polarized electron in a ferromagnet would be in a superposition of spin-mixed states, and this superposition can evolve toward an unpolarized state. Thus, spin mixing provides an intrinsic mechanism of spin relaxation in a ferromagnet. Further, spin mixing may impact charge-to-spin conversion in magnetic tunnel junctions [9] and spin-orbit related transport properties, such as the anomalous and planar Hall effects [10]. The future may also see refinements to FMR theory to more fully incorporate electronic structure and correlation effects that can be used to design and analyze spectroscopy experiments.

Andrew D. Kent: Department of Physics, New York University, New York, NY, USA

\section{REFERENCES}

1. C. Kittel, "On the gyromagnetic ratio and spectroscopic splitting factor of ferromagnetic substances," Phys. Rev. 76, 743 (1949).

2. J. M. Shaw et al., "Quantifying spin-mixed states in ferromagnets," Phys. Rev. Lett. 127, 207201 (2021).

3. M. B. Stearns, "Simple explanation of tunneling spin-polarization of Fe, $\mathrm{Co}$, Ni and its alloys," J. Magn. Magn. Mater. 5, 167 (1977).

4. J. C. Slonczewski, "Conductance and exchange coupling of two ferromagnets separated by a tunneling barrier," Phys. Rev. B 39, 6995 (1989).

5. A. Brataas et al., "Current-induced torques in magnetic materials," Nat. Mater. 11, 372 (2012).

6. J. Stöhr, "Exploring the microscopic origin of magnetic anisotropies with $\mathrm{x}$-ray magnetic circular dichroism (XMCD) spectroscopy," J. Magn. Magn. Mater. 200, 470 (1999).

7. C. T. Chen et al., "Experimental confirmation of the x-ray magnetic circular dichroism sum rules for iron and cobalt," Phys. Rev. Lett. 75, 152 (1995).

8. J. M. Shaw et al., "Precise determination of the spectroscopic 
g-factor by use of broadband ferromagnetic resonance spectroscopy,” J. Appl. Phys. 114, 243906 (2013).

9. J. C. Slonczewski and J. Z. Sun, "Theory of voltage-driven current and torque in magnetic tunnel junctions," J. Magn.
Magn. Mater. 310, 169 (2007).

10. C. Safranski et al., "Planar Hall driven torque in a ferromagnet/nonmagnet/ferromagnet system," Phys. Rev. Lett. 124, 197204 (2020). 